\title{
Towards a Quantitative Understanding in Electron Tomography
}

\author{
C. Kübel*, M. Godehardt**, R. Cieslinski***, S. Rozeveld***
}

* Forschungszentrum Karlsruhe, Institute for Nanotechnology, 76344 Eggenstein-Leopoldshafen, Germany

** Fraunhofer Insitute for Industrial Mathematics (ITWM), Fraunhofer-Platz 1, 67663 Kaiserslautern, Germany

*** The Dow Chemical Company, Venture \& Core R\&D, Analytical Sciences Laboratory, 1897 E. Building, Midland, MI 48667

State-of-the-art electron tomography is now a versatile tool for visualizing morphologies and particle distributions in $3 \mathrm{D}$ for a wide range of applications. Some prominent examples come from such diverse areas as catalysis [1], semiconductor materials [1,2], block copolymers [3], polymer composites [4] and biological applications [5].

In addition to improving the resolution in $3 \mathrm{D}$, one of the major aims in (electron) tomography is to move from purely qualitative imaging towards fully quantifiable results. In most cases, an ideal reconstruction of the $3 \mathrm{D}$ volume with noise limiting the image segmentation process is assumed for the quantification. Therefore, the implicit assumption is that in the absence of noise and with a complete tilt-series, identical materials within a sample should result in identical intensities in the reconstructed volume. However, our recent experimental results on different high-contrast data sets clearly show that this assumption is not valid. The reconstructed intensities are strongly dependent on the feature size and vary by 1-2 orders of magnitude for the same material within one reconstruction. This effect is present with SIRT and with WBPJ, however the exact dependency varies for both of them. These experimental findings have been confirmed using simulations assuming perfect projection conditions. These results and their strong implications for particle detection and quantification of any tomographic data will be discussed.

\section{References}

[1] C. Kübel et al., Microsc. Microanal., 11(5) (2005), 378-400.

[2] C. Kübel et al in '8th International Workshop of Stress-Induced Phenomena in Metallization', edited by E. Zschech, K. Maex, P.S. Ho, H. Kawasaki, T. Nakamura, AIP Conference Proceedings 817, (2006) page 223-228, American Institute of Physics, Melville, New York.

[3] V.H. Mareau et al., Macromolecules, 40(25) (2007), 9032-9039.

[4] H. Nishioka et al., Composite Interfaces 13 (7) (2006), 589-603.

[5] M. Beck et al., Nature 449 (2007) 611-615. 

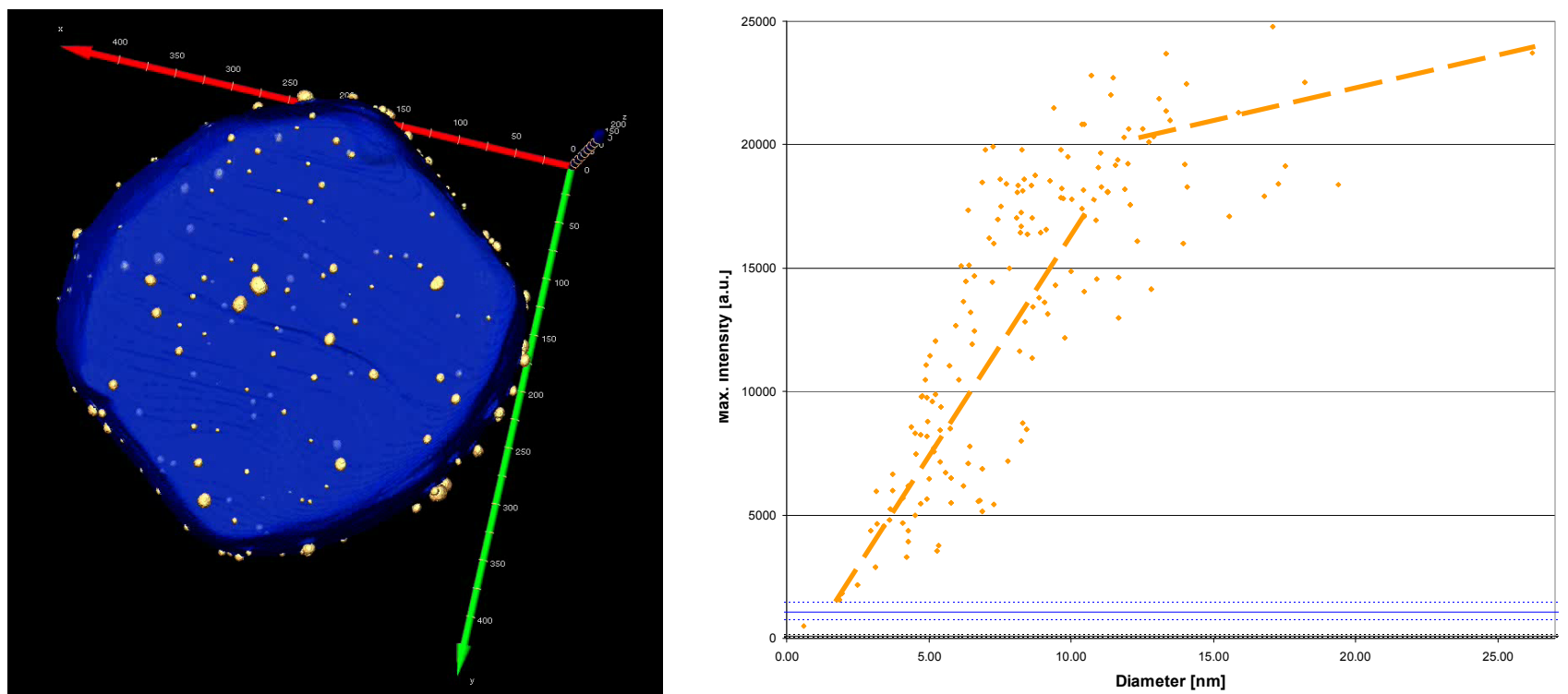

FIG. 1. Surface rendering of metal catalyst nanoparticles supported on a mesoporous silicate. The quantitative analysis of the reconstructed image intensities reveals a strong feature size dependence.
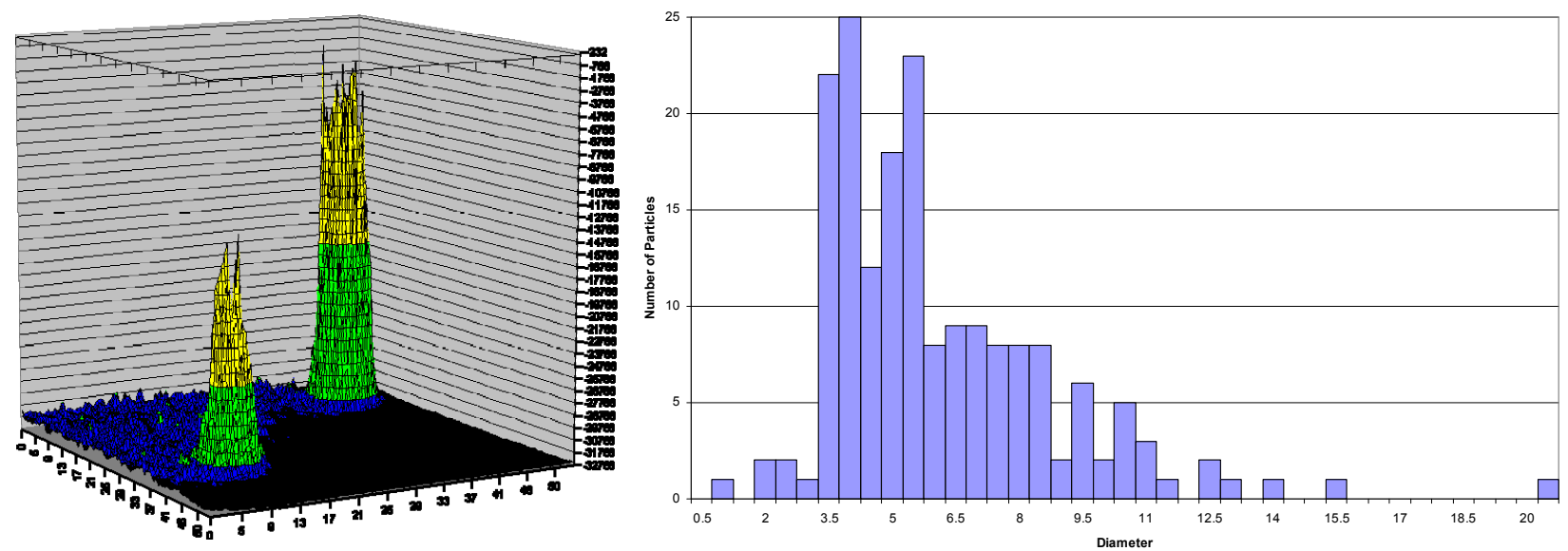

FIG. 2. The image segmentation was performed by local FWHM segmentation of every single particle resulting in a reliable particle size distribution. 